\title{
Strong-field ionization of molecular iodine traced with XUV pulses from a free-electron laser
}

\author{
Maria Krikunova, ${ }^{*}$ Theophilos Maltezopoulos, Philipp Wessels, Moritz Schlie, Armin Azima, Thomas Gaumnitz, \\ Thomas Gebert, Marek Wieland, and Markus Drescher \\ Institut für Experimentalphysik, Universität Hamburg, and Center for Free-Electron Laser Science CFEL, \\ Luruper Chaussee 149, 22761 Hamburg, Germany \\ (Received 12 July 2012; published 25 October 2012)
}

\begin{abstract}
Ultrafast dynamics of a molecular wave packet created by a strong 120 -fs near-infrared $(800 \mathrm{~nm})$ laser pulse in iodine has been probed by synchronized 13.4-nm, 35-fs extreme-ultraviolet pulses delivered by the free-electron laser facility in Hamburg, FLASH. The kinetic energy release of the multiply charged ionic fragments reveals three essential steps of strong-field-induced molecular fragmentation dynamics: (i) The creation of $\mathrm{I}_{2}{ }^{2+}$ and $\left(\mathrm{I}_{2}{ }^{2+}\right)^{*}$ molecular ions proceeds within $(75 \pm 15)$ fs full-width-at-half-maximum. (ii) With the onset of the $\mathrm{I}_{2}{ }^{2+}$ fragmentation the probability to lose a further electron within the same optical laser pulse rises with increasing $\mathrm{I}^{+}-\mathrm{I}^{+}$internuclear separation and reaches its maximum after $\sim 30 \mathrm{fs}$ with respect to the pulse maximum. (iii) Charge separation into the $\mathrm{I}_{2}{ }^{2+} \rightarrow \mathrm{I}^{2+}+\mathrm{I}$ dissociative channel with an asymmetric charge distribution is completed after $(121 \pm 22)$ fs.
\end{abstract}

DOI: 10.1103/PhysRevA.86.043430

PACS number(s): $33.80 . \mathrm{Rv}, 42.50 . \mathrm{Hz}, 32.80 . \mathrm{Fb}, 41.60 . \mathrm{Cr}$

\section{INTRODUCTION}

Intense light pulses can produce highly excited nonequilibrium states of matter within femtoseconds (fs). Short pulsed laser systems within the visible and near-infrared (NIR) region provide an electric field strength sufficient to suppress the outer barrier of atoms or molecules to the energy level of valence electrons. Diatomic molecules exposed to laser fields with an intensity exceeding $10^{13} \mathrm{~W} / \mathrm{cm}^{2}$ undergo multielectron dissociative ionization, leading to explosion of the molecule into ionic fragments with a characteristic kinetic energy release (KER) [1-4]. The nomenclature $\left(Q_{1}, Q_{2}\right)$ is widely used to describe each fragmentation channel of a diatomic molecule, where $Q_{1}$ and $Q_{2}$ are the corresponding charge states of the atomic ion.

Within other proposed mechanisms for dissociative ionization [4,5] the electron localization and enhanced ionization model [6-9] has proven to provide an appropriate explanation of experimental results [5,8,10-12]. For a molecule at the equilibrium internuclear distance $\left(R_{e}\right)$, the first ionization step occurs, like in the atomic case, by the ionization of one or more electrons. The singly or multiply charged molecular ion then starts to dissociate. With rising internuclear separation $R$ the probability of losing further electrons increases and reaches its maximum (enhanced ionization) at some critical internuclear separation $R_{\mathrm{cr}}$ [6,7]. A combination of fieldinduced strong coupling between charge resonance states and electron localization is thought to be responsible for the enhanced ionization [6,7]. Finally, the charges become separated on the corresponding atomic ions.

The enhanced ionization concept requires, however, that the time needed by the molecular ion to reach the critical internuclear separation $T_{\mathrm{cr}}$ has to be considerably shorter than the laser-pulse duration. Typically, $R_{\mathrm{cr}}$ is about a factor of 2 larger than the equilibrium internuclear separation $R_{e}[6,8,13]$.

\footnotetext{
*Institut für Optik und Atomare Physik, Technische Universität Berlin, Hardenbergstr. 36, EW 3-1, 10623 Berlin, Germany: maria.krikunova@physik.tu-berlin.de
}

For doubly charged molecular iodine $\mathrm{I}_{2}{ }^{2+}$ a Coulomb repulsion model predicts $T_{\mathrm{cr}} \sim 110 \mathrm{fs}$ [6] in correspondence with a first experimental finding of $T_{\mathrm{cr}} \sim 166 \mathrm{fs}$ [14]. Experiments $[15,16]$ have shown that iodine can be ionized up to $\mathrm{I}_{2}{ }^{13+}$, but due to much shorter pulse durations of $\sim 30$ fs employed there, all charge states were effectively formed at internuclear separations less than $R_{\mathrm{cr}}$. For the lightest diatomic molecular ion $\mathrm{H}_{2}{ }^{+}$(and $\mathrm{D}_{2}{ }^{+}$) the enhanced ionization region was also resolved experimentally at two critical internuclear separations [17], in agreement with theoretical predictions [7].

In the current study, we intend to disentangle the essential steps of strong-field-induced molecular fragmentation dynamics. The experimental challenge is to access the dedicated dynamics within the exciting NIR pulse with a short temporal gate. We use a NIR pulse of 120-fs full-width-at-halfmaximum (FWHM) duration at $1.6 \times 10^{14} \mathrm{~W} / \mathrm{cm}^{2}$ intensity to induce multielectron dissociative ionization and an intense ultrashort 35-fs FWHM extreme-ultraviolet (XUV) pulse from the free-electron laser (FEL) facility in Hamburg (FLASH) as a probe.

Figures 1(a)-1(c) show the principle of our experimental approach. The method relies on the quite different dynamics initiated by irradiation of $\mathrm{I}_{2}$ with the XUV pulse, as it has been shown in our previous study [18]. The 92-eV XUV pulse preferably interacts with the $4 d$ level of the atomic core [19]. The $4 d^{-1}$ vacancy is refilled within a few femtoseconds [20] preferably via single Auger decay (A1). Thus, the action of the XUV radiation on a particular molecular or atomic charge state $Q$ (populated by the NIR pulse) results in the quasi-instant (on the experimental time scale) increase of the charge state by at least two $(Q+2)$ as shown in Fig. 1(a). Within heavy molecular ions like iodine this additional charge will always be distributed between both atoms prior to fragmentation [18,21]. In this case, the NIR-induced fragmentation channel $\left(Q_{1}, Q_{2}\right)_{\text {NIR }}$ will be transferred by the XUV to $\left(Q_{1}+1, Q_{2}+1\right)_{\mathrm{NIR}+\mathrm{XUV}}$. However, as soon as the valence electrons become localized on the particular atomic core [Fig. 1(b)], the inner potential barrier prohibits electron exchange and the additional charge created by the XUV ionization will also become localized, i.e., a $Q_{1}$ charge state will be transferred to $Q_{1}+2$. 
(a) NIR and XUV simultaneously

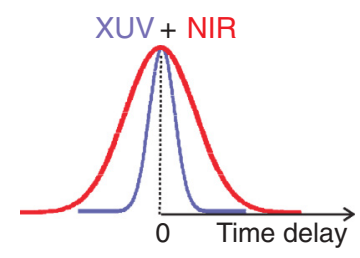

molecular ion (b) XUV later

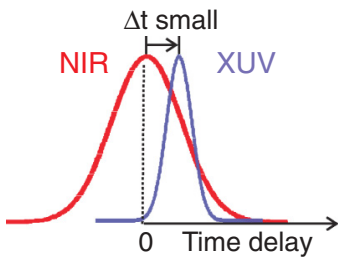

molecular ion with localized valence electrons (c) XUV later

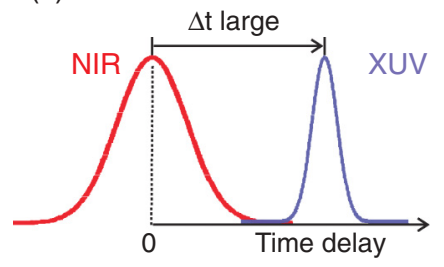

completed fragmentation, atomic fragments
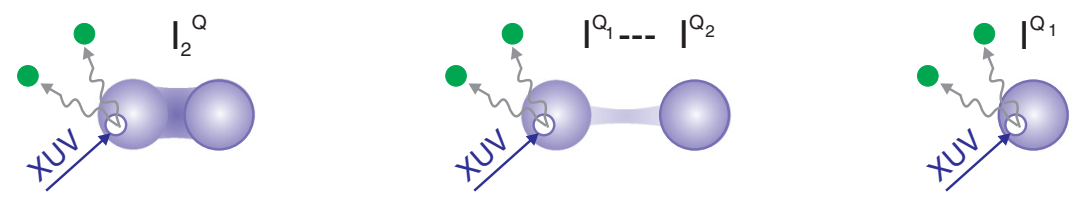

$\mathrm{I}^{\mathrm{Q}_{2}}$

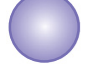

upon XUV ionization with subsequent Auger decay

$$
\begin{aligned}
& \mathrm{I}_{2}^{\mathrm{Q}+2} \\
& \left.\right|^{Q_{1}+2}---\left.\right|^{Q_{2}} \\
& l^{Q_{1}+2} \\
& \mathrm{I}^{\mathrm{Q}_{2}} \\
& \text { lonic fragments: } \\
& \mathrm{I}^{\mathrm{Q}_{1}+1}, \mathrm{l}^{\mathrm{Q}_{2}+1} \\
& I^{Q_{1}+2}, I^{Q_{2}} \\
& \mathrm{I}^{\mathrm{Q}_{1}+2}, \mathrm{I}^{\mathrm{Q}_{2}} \\
& \left(Q_{1}+1, Q_{2}+1\right)_{\mathrm{NIR}+\mathrm{XUV}} \\
& \left(Q_{1}+2, Q_{2}\right)_{N I R+X U V} \\
& \left(Q_{1}, Q_{2}\right)_{\text {NIR }}
\end{aligned}
$$

FIG. 1. (Color online) Principle of the NIR-pump-XUV-probe experiment to reveal multielectron dissociative ionization dynamics. The time-delay zero is defined as the coincidence of the NIR and XUV pulse maxima. Note that at large time delays (c) the XUV pulse hits the target after the molecular fragmentation is completed, i.e., the XUV-induced increase of charge $Q_{1}+2$ will not contribute to the increase of the KER.

The XUV ionization with subsequent Auger decay thus provides a way to probe the current electron configuration within fragmenting molecules and to unveil three essential steps of strong-field-induced molecular fragmentation dynamics shown in Fig. 1: creation of a molecular ion (a), valence electron localization due to the onset of fragmentation (b), and charge separation (c). Momentum-resolved ion charge-state spectroscopy is used to identify certain fragmentation channels through a characteristic KER of ionic fragments and to follow their evolution as a function of the NIR-pump-XUV-probe time delay. The interpretation of the experimental results is mainly focused on charge states $\geqslant 3$, as these states were not observed in our previous study of the collective dynamics induced by the XUV and a weak, i.e., below the threshold for dissociative ionization, NIR field [18]. Thus, these new fragmentation channels can unambiguously be ascribed to the channels produced by the dissociative ionization in a strong NIR field.

\section{EXPERIMENT}

A detailed description of the experimental setup can be found in Refs. [18,22]. Briefly, the experiment was performed at beamline BL1 of the FLASH facility operated at a photon energy near $92 \mathrm{eV}$ (13.4 nm wavelength) and at a $10-\mathrm{Hz}$ repetition rate in single bunch mode [23]. A mode-locked Ti:sapphire laser system (800-nm wavelength, 120-fs FWHM pulse duration, and pulse energy of $2 \mathrm{~mJ}$ ) provided by the FLASH facility [24] was electronically synchronized to the
1.3-GHz master clock of the accelerator. In the interaction region of the experiment the NIR laser beam was focused to $60-\mu \mathrm{m}$ FWHM and was overlapped with a focused XUV pulse of about $150-\mu \mathrm{m}$ FWHM spot size. The average XUV pulse duration was about 35-fs FWHM [25]. This results in an intensity in the target of about $1.6 \times 10^{14} \mathrm{~W} / \mathrm{cm}^{2}$ and $8 \times$ $10^{11} \mathrm{~W} / \mathrm{cm}^{2}$ for the NIR and XUV pulse, respectively.

Molecular iodine was expanded into the interaction region through a slightly heated gas nozzle and recollected with a liquid nitrogen cold trap. With operating gas nozzle the chamber pressure was kept in the range of $10^{-6}$ mbar. Momentum-resolved charge states of fragment ions were detected by a time-of-flight (TOF) spectrometer. It consists of an extraction and an acceleration region with electrical field strengths of $25 \mathrm{~V} / \mathrm{mm}$ and $500 \mathrm{~V} / \mathrm{mm}$, respectively, attached to a 62-cm-long field-free flight tube. A 2-mm-diameter aperture at the exit of the acceleration region was used to restrict the detection solid angle.

A methodological challenge of pump-probe experiments at free-electron lasers like FLASH are the arrival-time fluctuations of the XUV with respect to the NIR pulse in the order of a few hundred femtoseconds [26,27]. Specially developed tools to compensate the FEL timing jitter are reviewed in Ref. [28]. In the current study, a single-shot XUV/optical cross-correlator [29] for the arrival-time measurement was operated simultaneously with the pump-probe experiment. Momentum-resolved ion-charge-state spectra were collected at a fixed optical delay stage position while simultaneously 
measuring the XUV arrival time as described in Ref. [22]. Subsequently, the spectra were sorted according to the actual time delay within 30-fs time bins (about 200 single traces pro time bin) and averaged. As shown below, the resulting temporal resolution is better than the NIR pulse duration. The time-delay zero is defined as the maximum overlap between XUV and NIR pulses. It was determined with an accuracy of \pm 11 fs (95\% confidence bounds) from the fit of a transient $\mathrm{Xe}^{3+}$ yield and, additionally, of a transient $\mathrm{I}^{2+}$ ion yield within a $(2,1)$ channel as discussed in Refs. [18,22].

\section{EXPERIMENTAL RESULTS IN VIEW OF A CLASSICAL FIELD IONIZATION MODEL}

The current study is focused on the dynamics of the NIR-populated intermediate state $\mathrm{I}_{2}{ }^{2+}$, which is the precursor for $(1,1)_{\mathrm{NIR}}$ and $(2,0)_{\mathrm{NIR}}$ fragmentation channels. We are also looking for signatures of the $\mathrm{I}_{2}{ }^{3+}$ state, which undergoes further fragmentation through the $(2,1)_{\text {NIR }}$ channel. A very powerful approach to identify molecular intermediate states through their fragmentation channels is a coincident measurement with the reaction microscope (REMI) [30]. This technique represents the kinematically complete experiment as it provides information on full 3D momentum vectors of reaction fragments emitted in single events. Besides an obvious advantage of REMI, its application at low-rate radiation sources such as FELs represents an experimental challenge [30]. Alternatively, for simple diatomic molecules such as $I_{2}$ momentum resolved ion charge-state spectroscopy, as utilized in this work, may be used.

Figure 2 shows the momentum-resolved ion spectra induced by exclusive irradiation with XUV or NIR light. The assign-

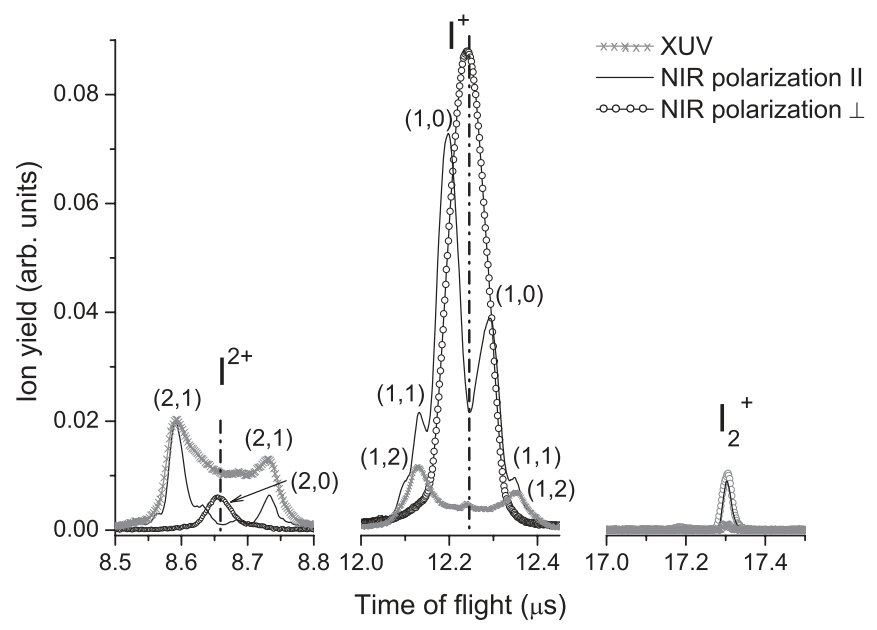

FIG. 2. Momentum-resolved ion yield spectra produced exclusively by XUV or NIR fields. Data were typically averaged over 80 single traces. The spectrometer efficiency is highest at zero KER (dash-dotted line in $\mathrm{I}^{+}$and $\mathrm{I}^{2+}$ part), dropping significantly with rising fragment momentum. This asymmetry with respect to the zero KER line is also seen in the higher detection efficiency for the ions starting with a momentum in spectrometer direction (shorter TOF) with respect to ions with opposite momentum (longer TOF). Note that the effective suppression of $(1,1)$ and $(1,2) /(2,1)$ channels compared to $(1,0)$ and $(2,0)$ channels by changing the NIR polarization with respect to the detector axis. ment of the fragmentation channels is based on their characteristic KER. Besides the molecular ion $\mathrm{I}_{2}{ }^{+}$, each channel in Fig. 2 exhibits two peaks in the ion yield spectra, one with shorter and one with longer time-of-flight (TOF), corresponding, respectively, to ions initially flying forward (fw) and backward (bw) with respect to the detector. The KER is calculated from the TOF difference between the bw and fw ion peaks.

We also use the sensitivity of multielectron dissociative ionization to the polarization direction of the NIR field to select and identify certain channels. In the intense laser field the ionization rate of $\mathrm{I}_{2}$ strongly depends on the orientation of the molecular axis with respect to the polarization direction as proven by the angular anisotropy in fragment distributions $[11,13]$ and supported by calculations based on a field ionization, Coulomb explosion model [9]. Calculations performed for aligned molecules [9] show that, under our NIR intensity conditions $\left(1.6 \times 10^{14} \mathrm{~W} / \mathrm{cm}^{2}\right)$, the ionization to the $\mathrm{I}_{2}{ }^{3+}$ state at $R_{e}$ can only be achieved in a fraction of molecules with their molecular axis oriented parallel to the electrical field vector. On the contrary, $\mathrm{I}_{2}{ }^{2+}$ can be created independently on the orientation of the molecular axis. In this context it is important that the $\mathrm{I}_{2}{ }^{2+}$ state has a $(2,0)_{\text {NIR }}$ and a $(1,1)_{\text {NIR }}$ dissociation limit. While the fragment distribution within the $(1,1)_{\text {NIR }}$ channel is strongly anisotropic with highest ion yield in the direction parallel to the electrical field vector, the $(2,0)_{\text {NIR }}$ channel reveals relatively low angle anisotropy $[11,13]$. Therefore, in the current study the signatures of $(1,1)_{\text {NIR }}$ and $(2,1)_{\text {NIR }}$ fragmentation channels can be substantially suppressed against the $(2,0)_{\text {NIR }}$ channel by rotating the NIR polarization perpendicular to the detection axis and by using an aperture within the time-of-flight spectrometer, which restricts the acceptance angle to about $\sim 15^{\circ}$ (compare spectra in Fig. 2 for NIR polarization parallel and perpendicular with respect to the detector axis).

Section III is organized in the following way: In Sec. III A we consider the temporal evolution of maximal irradiation intensity within the NIR pulse envelope in the framework of the classical field ionization approach introduced in Ref. [8,9]. The main idea is to predict the time (with respect to the NIR pulse maximum) of the creation of the $\mathrm{I}_{2}{ }^{2+}$ and $\mathrm{I}_{2}{ }^{3+}$ molecular wave packets. The dynamics of the $\mathrm{I}_{2}{ }^{2+}$ and $\mathrm{I}_{2}{ }^{3+}$ transient states are discussed in Secs. III B and IIIC, respectively. Notably, the experimental results of Sec. III B are obtained with perpendicular and of Sec. III C with parallel NIR polarization.

\section{A. Classical model of strong-field ionization as a function of the internuclear separation}

The field ionization, Coulomb explosion model introduced in Refs. [8,9] provides an estimation for the classical appearance intensity of each fragmentation channel. Briefly, for a molecule aligned parallel to the laser field $E$ the double-well potential $U$ (in $\mathrm{eV}$ ) seen by the outer electron can be approximated by

$$
U(x)=-B \frac{Q / 2}{|x+R / 2|}-B \frac{Q / 2}{|x-R / 2|}-e E(t) x,
$$

where $B=14.385 \mathrm{eV} \AA, Q=Q_{1}+Q_{2}$ is the sum of the atomic charge states, $x$ (in $\AA$ ) is the axial position of the electron, $R$ (in $\AA$ ) is the internuclear separation, and $E(t)$ is 
(a)

(b)

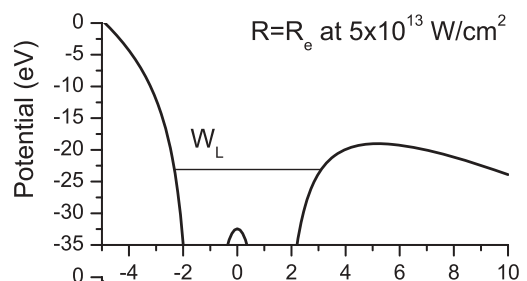

(c)

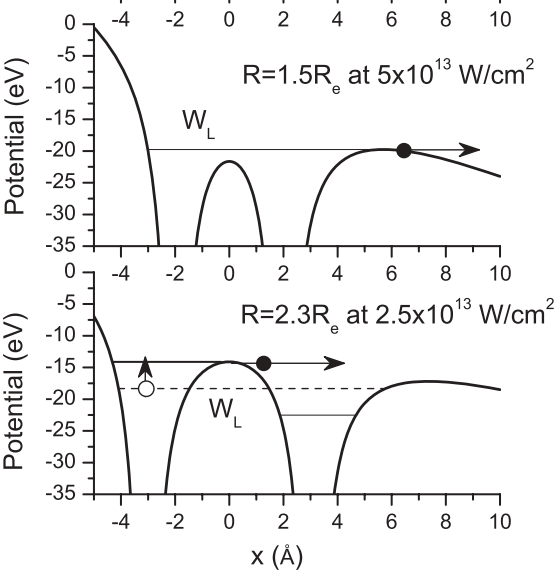

FIG. 3. Double-well potential for $\mathrm{I}_{2}{ }^{2+}$ at certain internuclear separations $R$ in a strong external field.

the instantaneous electric field strength of the NIR radiation (in $\mathrm{V} / \AA$ ). The energy level of the outer electron can be approximated by

$$
W_{L}=-\frac{V_{i 1}+V_{i 2}}{2}-B \frac{Q}{2 R},
$$

where $V_{i 1}$ and $V_{i 2}$ (in $\mathrm{eV}$ ) are known ionization potentials of both atomic ions. We consider a Gaussian function as a simple approximation for the irradiation intensity $I(t)$ within the NIR pulse envelope: $I(t)=I_{0} \exp \left(-\frac{t^{2}}{2 \sigma^{2}}\right)$, where $\sigma=51 \mathrm{fs}$ (120 fs FWHM) and $I_{0}=1.6 \times 10^{14} \mathrm{~W} / \mathrm{cm}^{2}$ (corresponding to $E_{0}=3.5 \mathrm{~V} / \AA$ ).

In the classical model, the electron can escape from the Coulomb potential of the ion, when the electrical field suppresses the outer barrier to the energy level $W_{L}$ of the electron. Figures 3(a) and 3(b) sketch the double-well potential calculated with Eq. (1) for the $\mathrm{I}_{2}{ }^{2+}$ state at two different internuclear separations, $R_{e}$ and $1.5 R_{e}$, respectively, but in the same external field of $2 \mathrm{~V} / \AA\left(5 \times 10^{13} \mathrm{~W} / \mathrm{cm}^{2}\right)$. Thus, at an intensity above the $5 \times 10^{13} \mathrm{~W} / \mathrm{cm}^{2}$ threshold, the $\mathrm{I}_{2}{ }^{2+} \rightarrow$ $\mathrm{I}_{2}{ }^{3+}$ transition becomes classically possible if the internuclear separation $R$ has reached at least $1.5 R_{e}$.

A further effect that lowers the minimum intensity for ionization is electron localization. For $\mathrm{I}_{2}$ at an internuclear separation longer than $1.5 R_{e}$, the central barrier rises above the energy level $W_{L}$ of the electron leading to the electron localization on a particular atomic core [8,9]. Due to the Stark shift by the factor of $0.5 R E$, the electron energy level may rise up to the top of the central barrier, allowing over-the-barrier ionization at longer internuclear separations and weaker external fields, as shown in Fig. 3(c) for $R=$ $2.3 R_{e}$ and $1.3 \mathrm{~V} / \AA$.

Figure 4(a) (solid line) shows the temporal evolution of the maximal irradiation intensity $I(t)$ within the NIR pulse
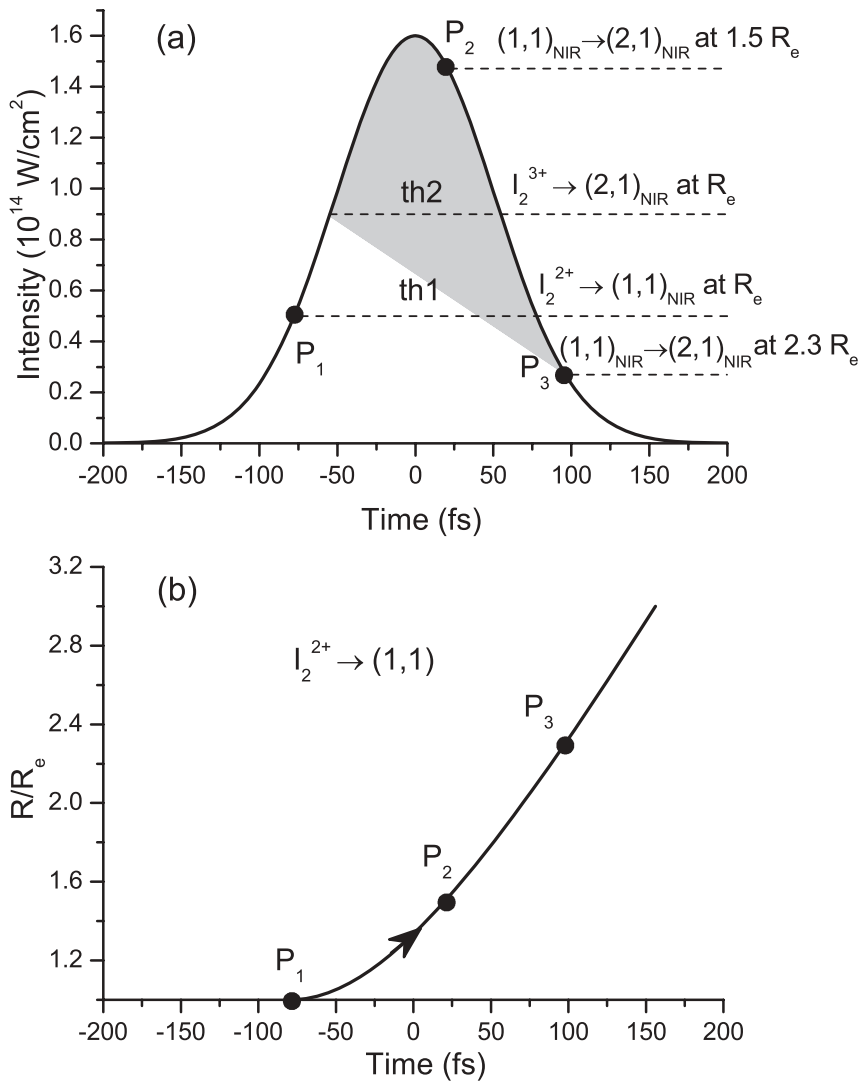

FIG. 4. (a) (Solid line) Evolution of $I(t)$ within the NIR pulse envelope, assuming a Gaussian pulse shape of 120-fs FWHM. (Dashed line) th1 and th2 indicate the threshold for the appearance of the $\mathrm{I}_{2}{ }^{2+}$ and $\mathrm{I}_{2}{ }^{3+}$ states, respectively, when the molecular ion is created at $R_{e}$. Gray shaded area indicates the temporal region within the NIR pulse, where the $\mathrm{I}_{2}{ }^{3+}$ at different internuclear separations can be produced. (b) The temporal evolution of the internuclear distance normalized to $R_{e}$ as calculated from the two-point charge model. We assume that the $\mathrm{I}_{2}{ }^{2+}$ state starts to dissociate at -80 fs [point $\mathrm{P}_{1}$ in panel (a)].

envelope. Above the thresholds th1 and th2 (dashed lines), respectively, the electrical field is sufficient to create $\mathrm{I}_{2}{ }^{2+}$ and $\mathrm{I}_{2}{ }^{3+}$ at $R_{e}$ if the molecular axis is aligned along the polarization direction. As discussed in Ref. [9], the appearance intensity for a certain molecular cationic state increases as the angle between the electrical field vector and molecular axis increases. Since our measurements are performed on an ensemble of randomly oriented molecules, th1 and th2 represent the lowest appearance threshold for $\mathrm{I}_{2}{ }^{2+}$ and $\mathrm{I}_{2}{ }^{3+}$ ionization, respectively.

Let us assume that the $\mathrm{I}_{2}{ }^{2+}$ state is created at $-80 \mathrm{fs}$ (with respect to the NIR pulse maximum) indicated by point $\mathrm{P}_{1}$ in Fig. 4(a) and starts to dissociate $\mathrm{I}_{2}{ }^{2+} \rightarrow \mathrm{I}^{+}-\mathrm{I}^{+}$. The temporal evolution of the internuclear separation can be modeled by considering the Coulomb repulsion between two point charges and integrating the classical equations of motions like in Ref. [31]. Figure 4(b) shows the corresponding calculation of the time-dependent increase of the distance between recoiling $\mathrm{I}^{+}$point charges. At point $\mathrm{P}_{2}$ (at $20 \mathrm{fs}$ ) the internuclear separation increases to $\sim 1.5 R_{e}$. Because the maximal field amplitude in this region of the NIR pulse is higher than $2 \mathrm{~V} / \AA$, 


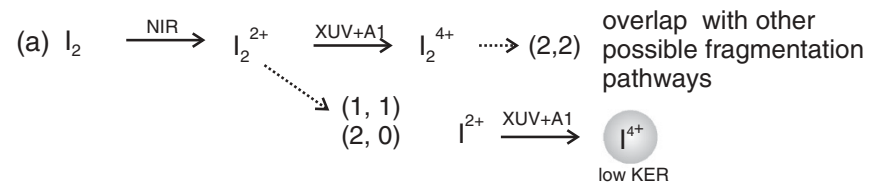

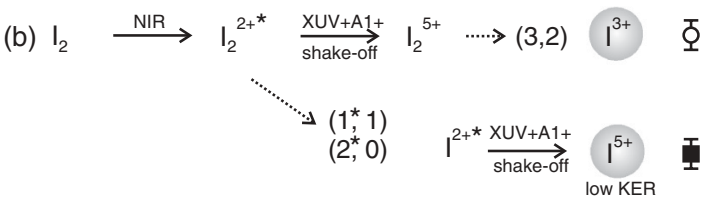

FIG. 5. Relevant fragmentation pathways leading to the detection of $\mathrm{I}^{3+}, \mathrm{I}^{4+}$, and $\mathrm{I}^{5+}$ transient ion yield (highlighted in gray). Symbols on the right side are the corresponding transient profiles plotted in Fig. 6 . The NIR polarization direction is perpendicular with respect to the detection axis.

a further ionization step $\mathrm{I}^{+}-\mathrm{I}^{+} \rightarrow \mathrm{I}^{2+}-\mathrm{I}^{+}$becomes possible, as discussed above [see also Fig. 3(b)]. Due to the electron localization which is expected to start after $20 \mathrm{fs}$ with respect to the NIR pulse maximum (corresponds to $>1.5 R_{e}$ as discussed in Ref. [8]) the $\mathrm{I}^{+}-\mathrm{I}^{+} \rightarrow \mathrm{I}^{2+}-\mathrm{I}^{+}$transition is possible up to the point $\mathrm{P}_{3}$ [the corresponding double-well potential is shown in Fig. 3(c)]. The gray shaded area of Fig. 4(a) indicates the temporal region within the NIR pulse, where the $\mathrm{I}_{2}{ }^{3+}$ transient states at different internuclear separations can be produced, e.g., at $R_{e}$ above th2, at $1.5 R_{e}$ around the point $\mathrm{P}_{2}$, or at $2.3 R_{e}$ around the point $\mathrm{P}_{3}$. Below $\mathrm{P}_{3}$ the electron energy level $W_{L}$ is below the inner barrier of the Coulomb potential, i.e., the electrical field strength is not sufficient for $\mathrm{a} \mathrm{I}^{+}-\mathrm{I}^{+} \rightarrow \mathrm{I}^{2+}-\mathrm{I}^{+}$transition.

According to the classical field ionization model, the temporal origin of $\mathrm{I}_{2}{ }^{2+}$ and $\mathrm{I}_{2}{ }^{3+}$ molecular cationic states is confined within the NIR pulse envelope [Fig. 4(a)]. The $\mathrm{I}_{2}{ }^{2+}$ state is expected to appear between -80 and $80 \mathrm{fs}$ [above threshold th1 in Fig. 4(a)]. The $\mathrm{I}_{2}{ }^{3+}$ can be created at different internuclear separations and should appear between -55 and $95 \mathrm{fs}$. Between 20 and $95 \mathrm{fs}$, the $\mathrm{I}^{2+}-\mathrm{I}^{+}$state with a localized valence electron configuration is expected.

\section{B. From the $\mathbf{I}_{2}{ }^{2+}$ molecular wave-packet creation to the $\mathrm{I}^{2+}+\mathrm{I}$ charge separation}

We now test the model of Sec. III A by analyzing the NIRXUV delay-dependent evolution of the ionic fragments with different final charge states $Q_{1}$ and the characteristic KER. According to Fig. 5, the presence of a transient molecular $\mathrm{I}_{2}^{2+}$ state could be detected through $(2,2)_{\mathrm{NIR}+\mathrm{XUV}}$ or $(3,2)_{\mathrm{NIR}+\mathrm{XUV}}$ fragmentation channels. Particularly, as already mentioned in the Introduction (Sec. I), an XUV pulse acting on the $\mathrm{I}_{2}^{2+}$ molecular ion will increase its charge state by at least two $(4 d$ ionization with subsequent Auger decay), leading to the $\mathrm{I}_{2}{ }^{4+}$ state. Moreover, the $\mathrm{I}_{2}{ }^{2+}$ state can also be electronically excited to $\left(\mathrm{I}_{2}{ }^{2+}\right)^{*}$ as found in Ref. [32]. This may additionally lead to shake-off of an excited electron on XUV ionization. Thus, the final molecular cationic state will be $\mathrm{I}_{2}{ }^{5+}$. Following Coulomb explosion of $\mathrm{I}_{2}{ }^{4+}$ and $\mathrm{I}_{2}{ }^{5+}$ molecular ions $\mathrm{I}^{2+}$ and $\mathrm{I}^{3+}$ ionic fragments with characteristic $(2,2)_{\mathrm{NIR}+\mathrm{XUV}}$ and $(3,2)_{\mathrm{NIR}+\mathrm{XUV}}$ KER should be detected, as sketched in Figs. 5(a) and 5(b), respectively.

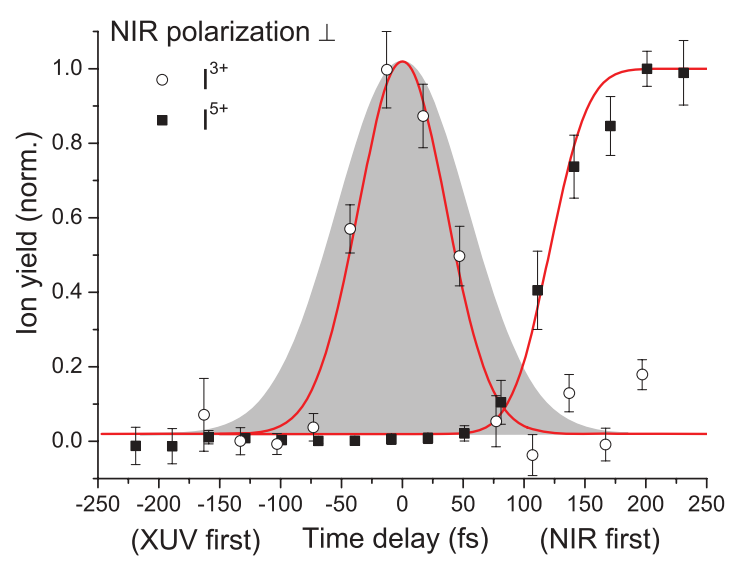

FIG. 6. (Color online) $\mathrm{I}^{3+}$ (open circles) and $\mathrm{I}^{5+}$ (closed squares) transient ion yield (background subtracted and normalized) as a function of time delay and the corresponding fits to the data (solid lines). Error bars are standard deviations. Gray filled curve represents the temporal overlap between XUV and NIR pulses approximated by the Gaussian profile of the 125-fs FWHM.

Observation of $\mathrm{I}^{2+}$ is prohibited due to the overlap with other channels [18]. Instead, the $\mathrm{I}^{3+}$ ionic fragments with a KER corresponding to the $(3,2)_{\mathrm{NIR}+\mathrm{XUV}}$ fragmentation channel are observed within the temporal overlap of NIR and XUV pulses (Fig. 6, open circles). Note that the signal appears within the region where the $\mathrm{I}_{2}{ }^{2+}$ intermediate state is expected [region above the threshold th1 in Fig. 4(a)]. The corresponding temporal profile was fitted with a Gaussian function of $(75 \pm 15)$ fs FWHM (Fig. 6, red solid line) and is considerably shorter than the temporal overlap between XUV and NIR pulses, represented in Fig. 6 by the gray filled area. The temporal overlap is approximated by a Gaussian profile of $(125 \pm 21)$ fs FWHM and is mainly determined by the NIR pulse duration [18,22]. Error bars represent $95 \%$ confidence bounds. The narrow temporal width of the $\left(\mathrm{I}_{2}{ }^{2+}\right)^{*}$ intermediate state reveals the time range within the NIR pulse envelope where the electrical field strength is sufficient for over-thebarrier ionization from the equilibrium internuclear separation. Our finding shows that the temporal origin of the $\mathrm{I}_{2}{ }^{2+}$ molecular cationic state is indeed confined within the NIR pulse envelope in correspondence with the current understanding of the multielectron dissociative ionization mechanism [6-9], as discussed in Sec. III A.

We are now looking for signatures in the ion yield spectra, where dissociation of $\mathrm{I}_{2}{ }^{2+}$ and $\left(\mathrm{I}_{2}{ }^{2+}\right)^{*}$ states is completed, i.e., the molecular wave packet has reached its final continuum state. Figure 5 summarizes two possible fragmentation pathways for $\mathrm{a} \mathrm{I}_{2}{ }^{2+}$ molecular cationic state with symmetric $(1,1)$ and asymmetric $(2,0)$ charge distribution. Correspondingly, for the $\left(\mathrm{I}_{2}{ }^{2+}\right)^{*}$ state the fragmentation pathways will be $\left(1^{*}, 1\right)$ and $\left(2^{*}, 0\right)$. In particular, in the detection direction perpendicular to the polarization vector, the yield in the $(1,1)$ fragmentation channel is suppressed compared to the $(2,0)$ channel with asymmetric charge distribution, as shown in Fig. 2 in correspondence with previous results [9,11]. The mechanism by which charge asymmetric channels such as $(2,0)$ are populated is currently not understood in detail [13]. 
As shown recently $[33,34]$, at the internuclear separation of $\sim 3.4 \AA$ the potential energy curve for the $\mathrm{I}_{2}{ }^{2+}$ state with the $(2,0)$ dissociation limit becomes essentially flat, i.e., the molecular wave packet has reached its asymptotic velocity. This would correspond to the appearance of two separated atomic cores $\mathrm{I}^{2+}+\mathrm{I}$ moving with a constant velocity along a flat potential energy curve. Note that after the charge separation $\mathrm{I}_{2}{ }^{2+} \rightarrow \mathrm{I}^{2+}+\mathrm{I}$ is completed, an additional charge created by the XUV pulse in the $\mathrm{I}^{2+}$ core will, in contrast to the I neutral core, not introduce an additional Coulomb repulsion force. Taking into account that the $(2,0)$ and $\left(2^{*}, 0\right)$ fragmentation channels can be identified through their relatively low KER $[14,34]$, the appearance of $\mathrm{I}^{4+}$ and $\mathrm{I}^{5+}$ ionic fragments with low KER is, thus, expected at long positive (NIR first) time delays (see Fig. 5).

The $\mathrm{I}^{4+}$ and $\mathrm{I}^{5+}$ fragment ions with low KER and similar transient behavior are indeed observed. The transient ion yield for the $\mathrm{I}^{5+}$ charge state with low KER is plotted in Fig. 6 (closed squares). The temporal profile was fitted with a smoothed step function (red solid line). The slope of the step function is comparable within $95 \%$ confidence bounds to the temporal width of the $(3,2)_{\mathrm{NIR}+\mathrm{XUV}}$ channel (Fig. 6, open circles) and is also narrower than one would expect from the $(125 \pm 21)$ fs width of the NIR-XUV temporal overlap. This suggests that both features originate from the same intermediate state: the former one at the instance of its creation by the NIR pulse $\left(\mathrm{I}_{2}{ }^{2+}\right)^{*}$ and the latter when the molecular wave packet has reached its final $\left(\mathrm{I}^{2+}\right)^{*}+\mathrm{I}$ state.

The $(121 \pm 22)$ fs shift of the inflection point toward positive time delays (NIR pulse first) provides an estimation for the time needed for $\left(\mathrm{I}^{2+}\right)^{*}$ and I charge separation. According to Refs. [33,34], the charge separation is expected to occur at the internuclear separation of $3.4 \AA$. Notably, $3.4 \AA$ is also close to the critical internuclear separation $R_{\mathrm{cr}}$, which is 1.3 to 2 times $R_{e}$ and $R_{e}=2.66 \AA$ (see Refs. [6,8,14]). Therefore, the determined characteristic time constant is also in close correspondence with $T_{\mathrm{cr}}$, the time needed for the molecular ion $\mathrm{I}_{2}{ }^{2+}$ to reach the critical internuclear separation predicted to $\sim 110$ fs in Ref. [6].

\section{Sequential $\mathrm{I}_{2}{ }^{2+} \rightarrow \mathrm{I}_{2}{ }^{3+}$ ionization by the same NIR pulse}

According to the enhanced ionization concept, the probability for a sequential ionization event $\mathrm{I}_{2}{ }^{2+} \rightarrow \mathrm{I}_{2}{ }^{3+}$ increases as the $\mathrm{I}_{2}{ }^{2+}$ state starts to dissociate. Under our experimental conditions, the $\mathrm{I}_{2}{ }^{3+}$ ionization state can be reached only in the fraction of molecules with the molecular axis occasionally oriented parallel to the electrical field vector, as discussed in Sec. III A and shown in Fig. 4(a) (gray filled area). Correspondingly, the ion yield spectra taken with the NIR polarization parallel to the detection axis are analyzed.

Figure 7 shows the momentum-resolved $\mathrm{I}^{3+}$ ion yield spectra at different time delays. Bold solid lines highlight a transient feature, which rises between -30 and 90 fs with maximum amplitude at about $30 \mathrm{fs}$. The appearance intensity threshold for this transient feature is about $9 \times 10^{13} \mathrm{~W} / \mathrm{cm}^{2}$ and correlates well with the appearance intensity threshold for the $(2,1)_{\text {NIR }}$ fragmentation channel in ion yield spectra taken with NIR pulses only (data not shown). Therefore, the transient feature in Fig. 7 corresponds to the signature

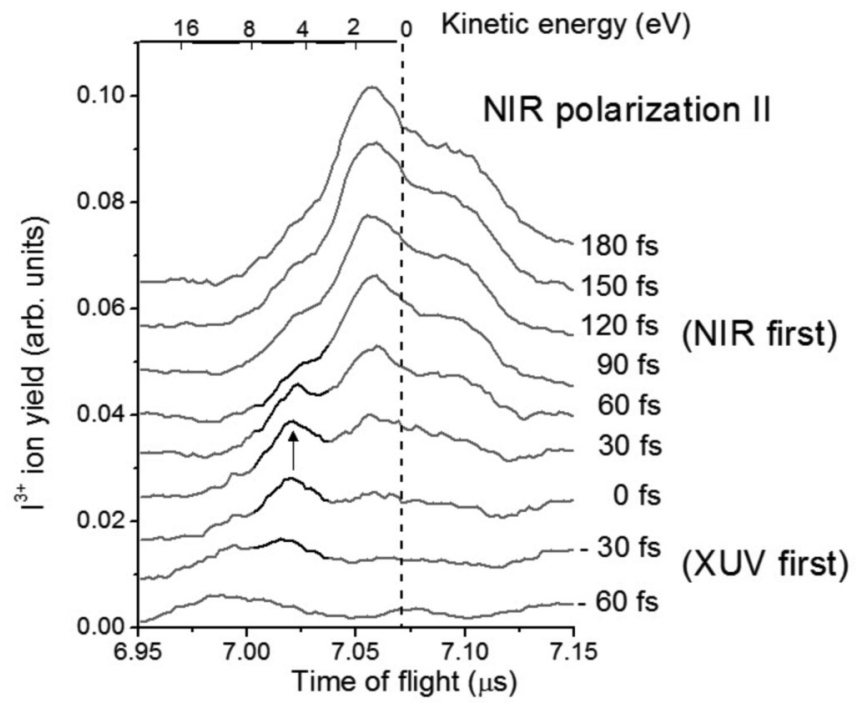

FIG. 7. Momentum-resolved $\mathrm{I}^{3+}$ ion yield spectra at certain time delays (solid lines). The scale on the top indicates the fragment kinetic energy (half of KER) for ions with the initial momentum on the spectrometer's axis. The transient feature (bold line) corresponds to a $(3,1)_{\text {NIR+XUV }}$ channel.

of the NIR-produced $\mathrm{I}_{2}{ }^{3+}$ intermediate state, which without XUV probe pulse will undergo fragmentation through the $(2,1)_{\text {NIR }}$ channel. Note that the pronounced spectral feature with low KER (close to the dashed line in Fig. 7) cannot be unambiguously assigned to a certain intermediate state. Temporal analysis of this feature indicates that it may be caused by several overlapping fragmentation channels.

The appearance of the $\mathrm{I}_{2}{ }^{3+}$ intermediate state in the $\mathrm{I}^{3+}$ part of the ion yield spectra can be understood as depicted in Fig. 8 . Essentially, the ionization potential of the $4 d$ core level of the $\mathrm{I}_{2}{ }^{3+}$ ionic ground state is about $\sim 10 \mathrm{eV}$ higher [35] than the XUV photon energy of $92 \mathrm{eV}$. This means that as long as the atomic cores are not completely separated, $\mathrm{I}_{2}{ }^{3+} \rightarrow \mathrm{I}^{2+}+\mathrm{I}^{+}$, the XUV pulse can interact only with valence electrons of the $\mathrm{I}_{2}{ }^{3+}$ state and the total charge increase will be only $Q+1$. Accordingly, after the interaction with the XUV probe pulse, the NIR-produced $\mathrm{I}_{2}{ }^{3+}$ transient state will be transferred to $\mathrm{I}_{2}{ }^{4+}$.

A further important factor is the valence electron configuration of the $\mathrm{I}_{2}{ }^{3+}$ intermediate state. On XUV ionization to the $\mathrm{I}_{2}{ }^{4+}$ state the most probable fragmentation pathway is the $(2,2)_{\text {NIR }+X U V}$ channel with symmetric charge distribution $[18,21]$. However, as soon as valence electrons become localized on a particular atomic core $\mathrm{I}^{2+}-\mathrm{I}^{+}$, the XUV ionization

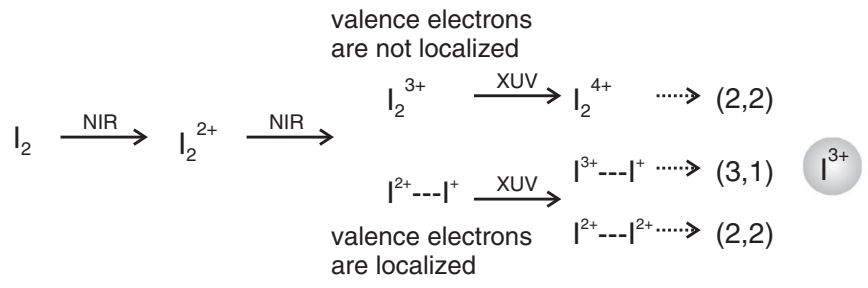

FIG. 8. Relevant fragmentation pathways leading to the detection of the transient $\mathrm{I}^{3+}$ ion yield (highlighted in gray) as shown in Fig. 7. 
will be atomiclike, i.e., the XUV pulse will interact with the $\mathrm{I}^{2+}$ or $\mathrm{I}^{+}$atomic ion. This will lead to the appearance of new fragmentation pathways $\mathrm{I}^{3+}-\mathrm{I}^{+} \rightarrow(3,1)_{\mathrm{NIR}+\mathrm{XUV}}$ and $\mathrm{I}^{2+}-\mathrm{I}^{2+} \rightarrow(2,2)_{\mathrm{NIR}+\mathrm{XUV}}$. Thus, the transient feature in the $\mathrm{I}^{3+}$ part of the ion yield spectra shown in Fig. 7 can be assigned to the $(3,1)$ fragmentation channel as summarized in Fig. 8. From a characteristic KER of $\sim 13 \mathrm{eV}$ (corresponds to the kinetic energy of $\sim 6.5 \mathrm{eV}$ as roughly estimated from Fig. 7), the fragmentation within this channel has started at the internuclear separation of $\sim 1.4 R_{e}$. According to the Coulomb explosion model [Fig. 4(a)] $1.4 R_{e}$ can be easily achieved within the NIR pulse envelope.

The temporal behavior of the transient feature confirms that the NIR-populated $\mathrm{I}^{2+}-\mathrm{I}^{+}$intermediate state with a localized valence electron configuration is confined within the NIR pulse envelope. The signal is restricted to the region of time delays between -30 and 90 fs with a signal maximum at about 30 fs (Fig. 7). A symmetric double-well Coulomb potential model predicts [8] that electron localization starts at about $1.5 R_{e}$, which fits well with $1.4 R_{e}$ estimated from our measurements. According to the model shown in Fig. 4(a) the $1.5 R_{e}$ internuclear separation should be achieved around point $\mathrm{P}_{2}$ (20 fs with respect to the NIR pulse maximum), which is also close to our experimental finding. Thus, the method reveals the region within the NIR pulse envelope where the $\mathrm{I}^{2+}-\mathrm{I}^{+}$intermediate state with a localized valence electron configuration is created. This finding shows that electron localization is essential for multielectron dissociative ionization in a strong laser field.

\section{SUMMARY}

We have studied the dynamics of a $\mathrm{I}_{2}{ }^{2+}$ molecular wave packet excited by a strong $800-n m$ NIR pulse. Three essential states of its evolution have been revealed: First, the temporal origin of the $\mathrm{I}_{2}{ }^{2+}$ molecular wave packet is confined to ( $75 \pm$ 15) fs FWHM within the 120-fs FWHM NIR pulse. Second, we have localized the region of enhanced ionization within the NIR pulse envelope. Under our experimental conditions the probability for the sequential ionization event $\mathrm{I}_{2}{ }^{2+} \rightarrow \mathrm{I}_{2}{ }^{3+}$ reaches its maximum after $\sim 30$ fs with respect to the NIR pulse maximum. In this case the valence electrons of the $\mathrm{I}^{2+}-\mathrm{I}^{+}$ state are localized on the particular ionic core. Third, at (121 \pm 22) fs the molecular wave packet $\mathrm{I}_{2}{ }^{2+}$ with asymmetric dissociation limit $\mathrm{I}^{2+}+\mathrm{I}$ has reached its final continuum state. Our results are understood in terms of a classical field ionization model and demonstrate the crucial role of valence electron localization for the subsequent ionization events within the same NIR pulse.

The experimental approach used here relies on the ability of XUV radiation to interrogate core levels of a particular ion and on the sensitivity of the subsequent Auger decay to the valence electron configuration. By using considerably shorter XUV pulses, it was possible to track dynamical details within the envelope of a longer NIR pulse. Due to different interaction mechanisms of pump and probe pulses with the target molecules, and the nonlinearity of the strong-field ionization, the temporal resolution is better than dictated by the convolution of pump and probe pulse profiles.

\section{ACKNOWLEDGMENTS}

This work was supported by the Bundesministerium für Bildung und Forschung (FSP-301) and the German Science Foundation (SFB 925). Nikolay M. Kabachnik is gratefully acknowledged for fruitful discussions and his continuing interest in this work. Finally, the authors thank the scientific and technical team of FLASH for making this work possible.
[1] L. J. Frasinski, K. Codling, P. Hatherly, J. Barr, I. N. Ross, and W. T. Toner, Phys. Rev. Lett. 58, 2424 (1987).

[2] K. Codling and L. J. Frasinski, J. Phys. B 26, 783 (1993).

[3] D. T. Strickland, Y. Beaudoin, P. Dietrich, and P. B. Corkum, Phys. Rev. Lett. 68, 2755 (1992).

[4] M. Schmidt, D. Normand, and C. Cornaggia, Phys. Rev. A 50, 5037 (1994).

[5] D. Normand and M. Schmidt, Phys. Rev. A 53, R1958 (1996).

[6] T. Seideman, M. Y. Ivanov, and P. B. Corkum, Phys. Rev. Lett. 75, 2819 (1995).

[7] T. Zuo and A. D. Bandrauk, Phys. Rev. A 52, R2511 (1995).

[8] J. H. Posthumus, A. J. Giles, M. R. Thompson, and K. Codling, J. Phys. B 29, 5811 (1996).

[9] J. H. Posthumus, J. Plumridge, M. K. Thomas, K. Codling, L. J. Frasinski, A. J. Langley, and P. F. Taday, J. Phys. B 31, L553 (1998).

[10] C. Cornaggia, J. Lavancier, D. Normand, J. Morellec, P. Agostini, J. P. Chambaret, and A. Antonetti, Phys. Rev. A 44, 4499 (1991).

[11] P. Dietrich, D. T. Strickland, M. Laberge, and P. B. Corkum, Phys. Rev. A 47, 2305 (1993).
[12] P. A. Hatherly, M. Stankiewicz, K. Codling, L. J. Frasinski, and G. M. Cross, J. Phys. B 27, 2993 (1994).

[13] H. Chen, L. Fang, V. Tagliamonti, and G. N. Gibson, Phys. Rev. A 84, 043427 (2011).

[14] E. Constant, H. Stapelfeldt, and P. B. Corkum, Phys. Rev. Lett. 76, 4140 (1996).

[15] G. N. Gibson, M. Li, C. Guo, and J. P. Nibarger, Phys. Rev. A 58, 4723 (1998).

[16] S. V. Menon, J. P. Nibarger, and G. N. Gibson, J. Phys. B 35, 2961 (2002).

[17] T. Ergler, A. Rudenko, B. Feuerstein, K. Zrost, C. D. Schroeter, R. Moshammer, and J. Ullrich, J. Phys. B 39, S493 (2006).

[18] M. Krikunova, T. Maltezopoulos, P. Wessels, M. Schlie, A. Azima, M. Wieland, and M. Drescher, J. Chem. Phys. 134, 024313 (2011).

[19] F. Comes, U. Nielsen, and W. Schwarz, J. Chem. Phys. 58, 2230 (1973).

[20] J. Cutler, G. Bancroft, and K. Tan, J. Chem. Phys. 97, 7932 (1992).

[21] B. Boo and N. Saito, J. Electron Spectrosc. Relat. Phenom. 127, 139 (2002). 
[22] M. Krikunova, T. Maltezopoulos, A. Azima, M. Schlie, U. Fruehling, H. Redlin, R. Kalms, S. Cunovic, N. M. Kabachnik, M. Wieland, and M. Drescher, New J. Phys. 11, 123019 (2009).

[23] K. Tiedtke et al., New J. Phys. 11, 023029 (2009).

[24] H. Redlin, A. Al-Shemmary, A. Azima, N. Stojanovic, F. Tavella, I. Will, and S. Duesterer, Nucl. Instrum. Methods A 635, S88 (2011).

[25] U. Fruehling, M. Wieland, M. Gensch, T. Gebert, B. Schuette, M. Krikunova, R. Kalms, F. Budzyn, O. Grimm, J. Rossbach, E. Ploenjes, and M. Drescher, Nat. Phot. 3, 523 (2009).

[26] S. Cunovic, N. Muller, R. Kalms, M. Krikunova, M. Wieland, M. Drescher, T. Maltezopoulos, U. Fruhling, H. Redlin, E. Plonjes-Palm, and J. Feldhaus, Appl. Phys. Lett. 90, 121112 (2007).

[27] P. Radcliffe, S. Duesterer, A. Azima, H. Redlin, J. Feldhaus, J. Dardis, K. Kavanagh, H. Luna, J. P. Gutierrez, P. Yeates, E. T. Kennedy, and J. T. Costello, Appl. Phys. Lett. 90, 131108 (2007).
[28] M. Drescher, U. Fruehling, M. Krikunova, T. Maltezopoulos, and M. Wieland, J. Phys. B 43, 194010 (2010).

[29] T. Maltezopoulos, S. Cunovic, M. Wieland, M. Beye, A. Azima, H. Redlin, M. Krikunova, R. Kalms, U. Fruehling, F. Budzyn, W. Wurth, A. Foehlisch, and M. Drescher, New J. Phys. 10, 033026 (2008).

[30] A. Rudenko, Y. H. Jiang, M. Kurka, K. U. Kuehnel, L. Foucar, O. Herrwerth, M. Lezius, M. F. Kling, C. D. Schroeter, R. Moshammer, and J. Ullrich, J. Phys. B 43, 194004 (2010).

[31] P. Dietrich, D. Strickland, and P. Corkum, J. Phys. B 26, 2323 (1993).

[32] J. P. Nibarger, M. Li, S. Menon, and G. N. Gibson, Phys. Rev. Lett. 83, 4975 (1999).

[33] L. Fang and G. N. Gibson, Phys. Rev. A 75, 063410 (2007).

[34] L. Fang and G. N. Gibson, Phys. Rev. A 81, 033410 (2010).

[35] V. Jonauskas, L. Partanen, S. Kucas, R. Karazija, M. Huttula, S. Aksela, and H. Aksela, J. Phys. B 36, 4403 (2003). 